\title{
Supporting Information: Electrically tunable all-PCM visible plasmonics
}

Kandammathe Valiyaveedu Sreekanth, ${ }^{\mathrm{a}, \mathrm{b}}$ Rohit Medwal, ${ }^{\mathrm{c}}$ Chandreyee M. Das, ${ }^{\mathrm{d}, \mathrm{e}}$ Manoj Gupta, ${ }^{\mathrm{a}}$

${ }^{b}$ Mayank Mishra, ${ }^{c}$ Ken-Tye Yong, d, e Rajdeep Singh Rawat, ${ }^{c}$ and Ranjan Singh* a, b

a. Division of Physics and Applied Physics, School of Physical and Mathematical Sciences, Nanyang Technological University, 21 Nanyang Link, Singapore-637371

b. Centre for Disruptive Photonic Technologies, The Photonic Institute, 50 Nanyang Avenue, Singapore-639798

c. Natural Sciences and Science Education, National Institute of Education, Nanyang Technological University, Singapore 637616, Singapore

d. School of Electrical and Electronic Engineering, Nanyang Technological University, Singapore, 639798

e. CINTRA CNRS/NTU/THALES, UMI 3288, Research Techno Plaza, 50 Nanyang Drive, Nanyang Technological University, Singapore, 637553

\section{Methods}

Sample fabrication. The Si and quartz substrates were pre-cleaned using acetone, ethanol, and deionized water prior to deposition. $\mathrm{Sb}_{2} \mathrm{Te}_{3}$ thin films were deposited using $\mathrm{RF}$ magnetron sputtering. The distance between the substrate and the target was kept $15 \mathrm{~cm}$ to achieve uniform deposition of thin films. The deposition was carried out at room temperature at a fixed RF power of 70 Watt under high purity argon (99.999\%) at a deposition pressure of $10 \mathrm{mTorr}$.

Microheater fabrication and Temperature calibration: The metallic microheater was fabricated by optical lithography. Thin film of Au with a thickness of $200 \mathrm{~nm}$ was thermally deposited over the entire area and followed by a lift-off process to leave behind the Au only in the patterned area. Thereafter, the microheater was subjected to the temperature calibration using Keithley 2450. A thermocouple was mounted at the center of the microheater, and two probes were used to apply the DC current on the heating element. The temperature was recorded as a function of the applied current. 
Nanograting fabrication: Focused (gallium)-ion beam (FEI Helios NanoLab 600) was used to fabricate nanograting metasurfaces. By using FIB with a beam current $\leq 15 \mathrm{pA}$ and dosage of 5 $\mathrm{mC} / \mathrm{cm}^{2}$, we milled through $30 \mathrm{~nm}$ thick $\mathrm{Sb}_{2} \mathrm{Te}_{3}$ thin film deposited on $\mathrm{Si}$ and quartz.

Ellipsometry characterizations. Variable-angle high-resolution spectroscopic ellipsometry (J.A. Woollam Co., Inc., V-VASE) was used to determine the thickness and the optical constants of $\mathrm{Sb}_{2} \mathrm{Te}_{3}$ thin films. To obtain the complex dielectric constants of $\mathrm{Sb}_{2} \mathrm{Te}_{3}$ thin films in both amorphous and crystalline phases, we deposited $50 \mathrm{~nm} \mathrm{Sb}{ }_{2} \mathrm{Te}_{3}$ thin film on silicon and quartz substrates and a Tauc-Lorentz model was used to fit the measured ellipsometry data. Since the "as deposited" $\mathrm{Sb}_{2} \mathrm{Te}_{3}$ thin films are in amorphous phase, we annealed at $150^{\circ} \mathrm{C}$ for $15 \mathrm{~min}$ to switch the phase from amorphous to polycrystalline. The polycrystalline $\mathrm{Sb}_{2} \mathrm{Te}_{3}$ thin film was fitted again using a Tauc-Lorentz oscillator model.

Prism coupling experiments. A custom-built angular surface plasmon resonance (SPR) spectroscopy set-up was used. We used a continuous wave $532 \mathrm{~nm}$ DPSS laser with a power of 5 $\mathrm{mW}$ and a beam spot size of $1 \mathrm{~mm}$ in diameter as the optical source. We performed the experiments for both TM and TE polarizations. The prism-coupling configuration is based on the well-known Kretschmann configuration, where the $50 \mathrm{~nm}$ thick $\mathrm{Sb}_{2} \mathrm{Te}_{3}$ thin film was directly deposited to the hypotenuse face of a right-angle prism coupler made from NLAF 36 glass (refractive index, 1.865) that was immobilized on a rotary translation stage. The intensity of the totally internally reflected light with the incident angle was collected and monitored through a high-precision optical power meter (Newport 2832C). The reflectance data were calibrated against a highly reflective surface. Reflectance measurement. The normal incidence reflectance measurements were performed using a microspectrophotometer (Jasco, MSV-5200) with a sampling domain size of $25 \mu \mathrm{m} \times 25 \mu \mathrm{m}$ and a numerical aperture of 0.7 . 
Numerical simulations. Angular reflectance data were simulated using transfer matrix method code written in MATLAB. Finite difference time domain (FDTD) method has been used to simulate the normal incidence reflectance spectra and intensity field distribution. The commercially available Lumerical FDTD software (lumerical.com) was used for this purpose. We considered a patterned (2D) $\mathrm{Sb}_{2} \mathrm{Te}_{3} /$ quartz system. In the 3D simulation, periodic boundary condition was used along $\mathrm{x}$ and $\mathrm{y}$ directions and perfectly matched layer (PML) boundary condition was used along $\mathrm{z}$ direction. In addition, plane wave source was used as the incident beam. Experimentally determined complex permittivities of $\mathrm{Sb}_{2} \mathrm{Te}_{3}$ thin film were used in the simulations.
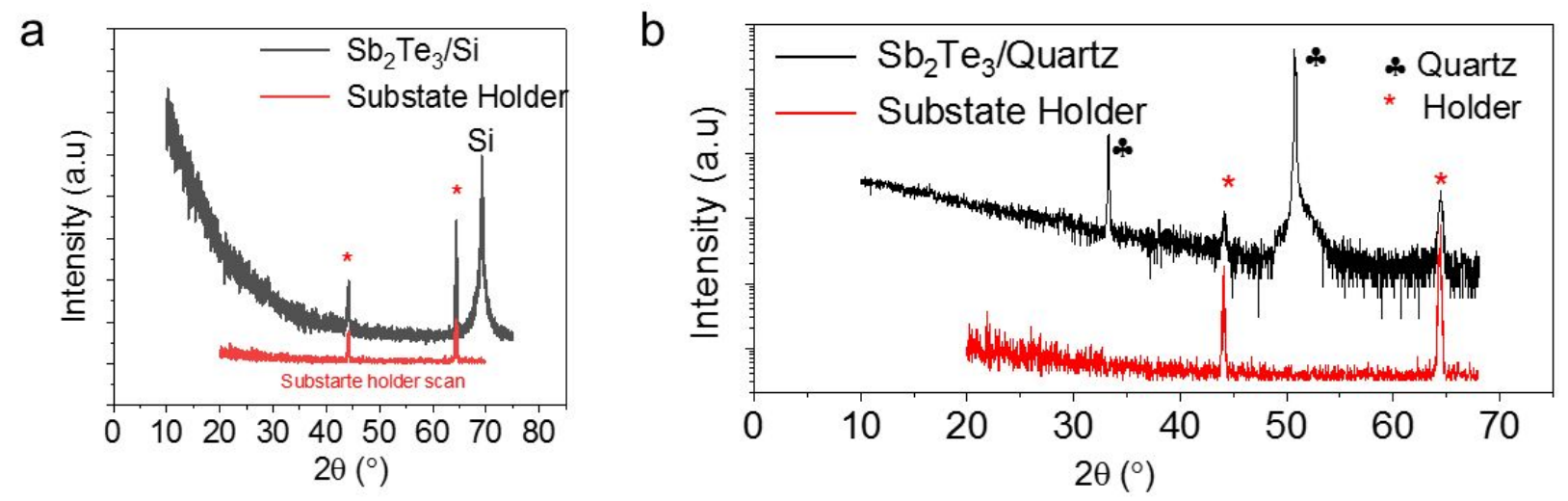

Figure S1. XRD spectrum of (a) $\mathrm{Sb}_{2} \mathrm{Te}_{3}$ thin film on Si substrate and (b) $\mathrm{Sb}_{2} \mathrm{Te}_{3}$ thin film on quartz substrate. 

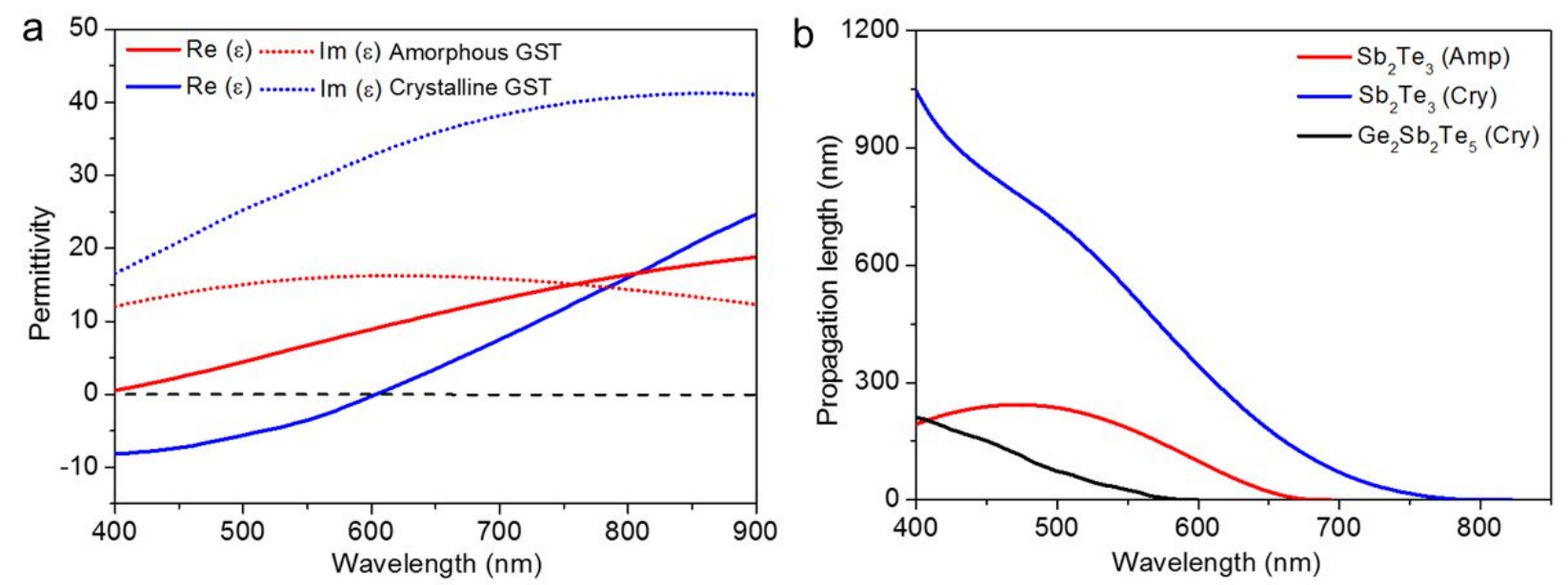

Figure S2. (a) Experimentally determined complex permittivities of $\mathrm{Ge}_{2} \mathrm{Sb}_{2} \mathrm{Te}_{5}$ thin film in both amorphous and crystalline phases. (b) Calculated propagation distance of $\mathrm{Sb}_{2} \mathrm{Te}_{3}$ and $\mathrm{Ge}_{2} \mathrm{Sb}_{2} \mathrm{Te}_{5}$.
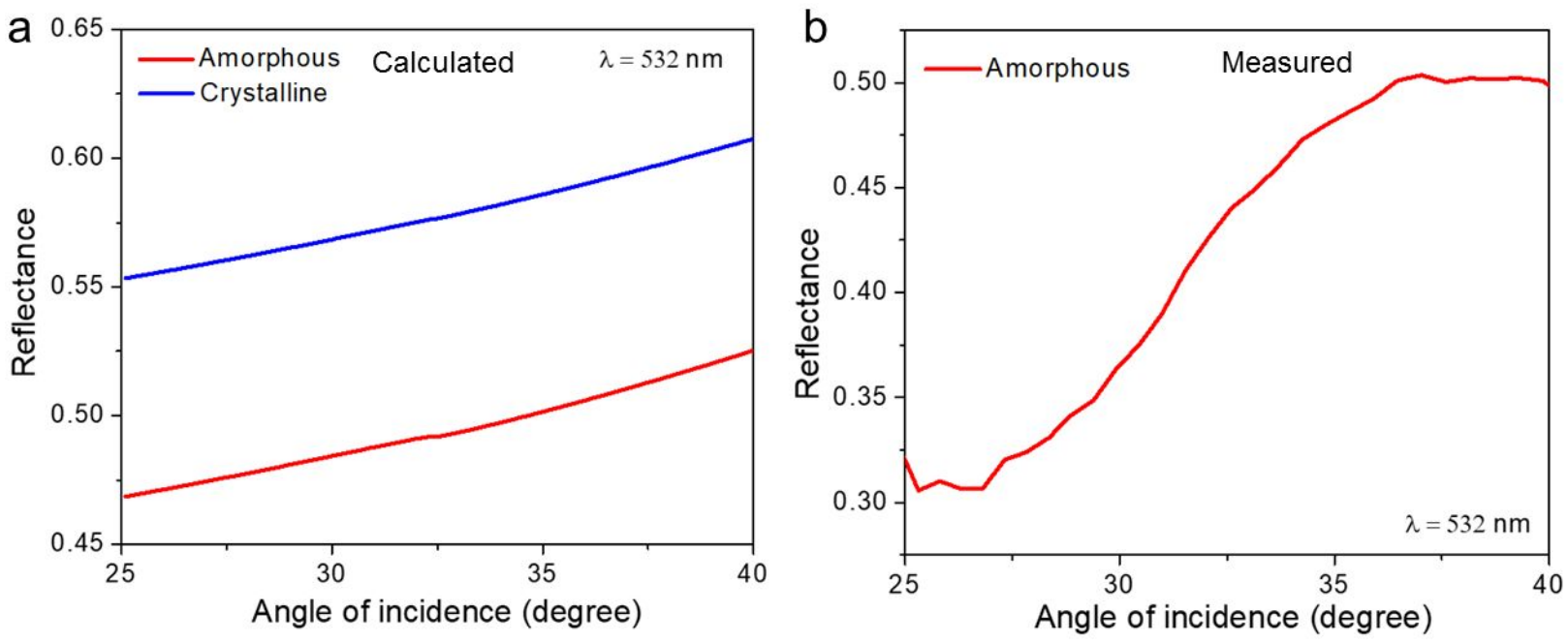

Figure S3. For TE polarization (a) Calculated reflectance as a function of incident angle at 523 $\mathrm{nm}$ for both phases of $\mathrm{Sb}_{2} \mathrm{Te}_{3}$ thin film (b) Measured reflectance as a function of incident angle at $523 \mathrm{~nm}$ for amorphous phase of $\mathrm{Sb}_{2} \mathrm{Te}_{3}$ thin film. 

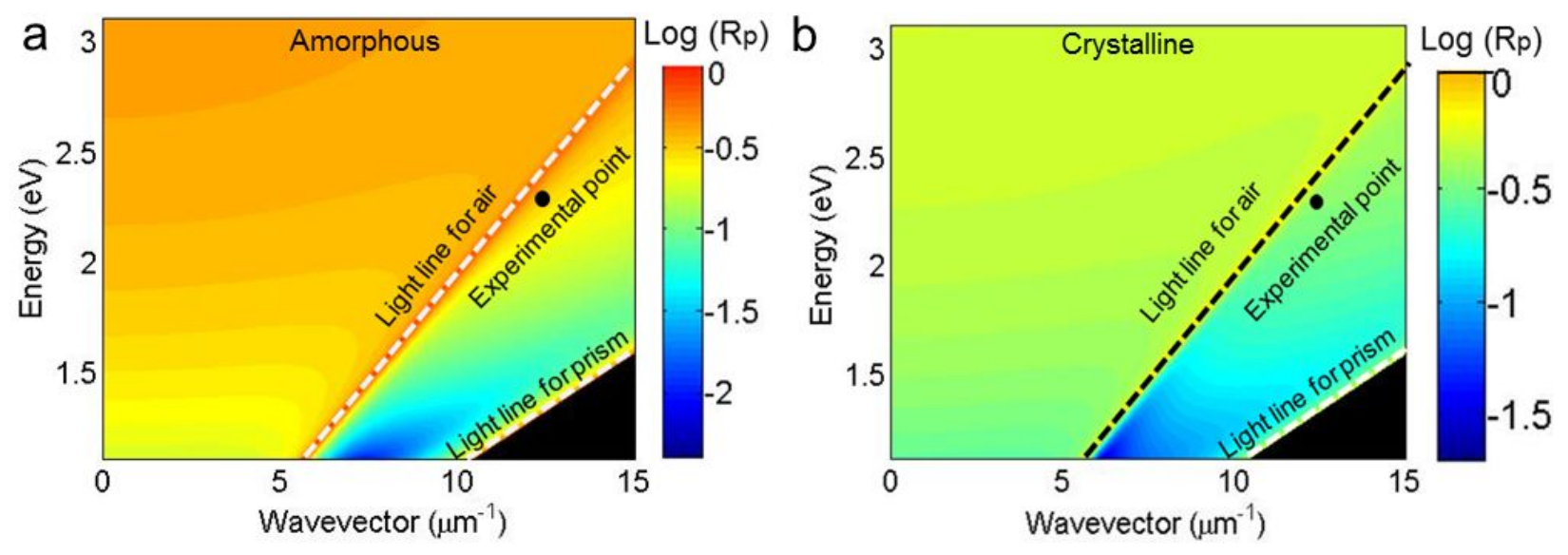

Figure S4. Calculated dispersion diagram for $\mathrm{TM}$ polarization when $\mathrm{Sb}_{2} \mathrm{Te}_{3}$ thin film is in (a) amorphous phase and (b) crystalline phase.

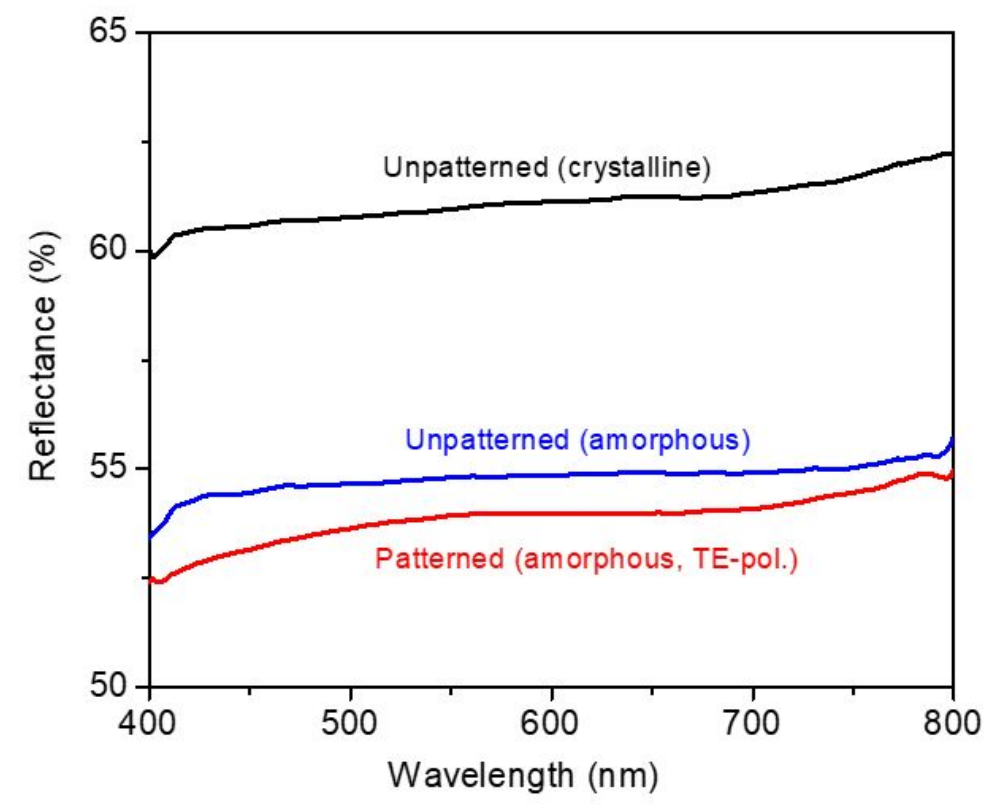

Figure S5. Reflectance spectrum of $\mathrm{Sb}_{2} \mathrm{Te}_{3}$ unpatterned film (amorphous and crystalline phases) and patterned $1 \mathrm{D}$ grating lines for TE polarization. 

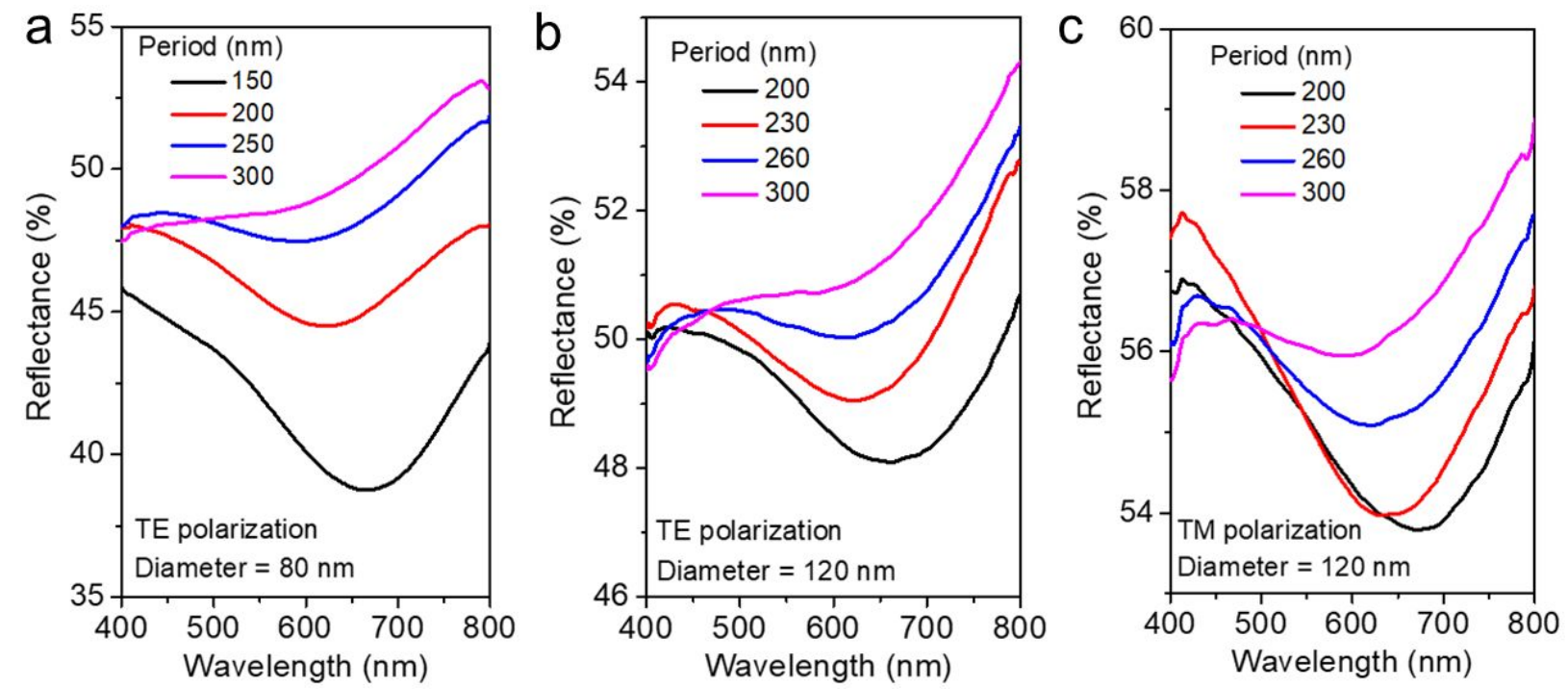

Figure S6. Measured reflectance spectra of 2D diffraction grating with different periods (a) TE polarized spectra of nanoholes with diameter $80 \mathrm{~nm}$. Reflection spectra of nanoholes with diameter $120 \mathrm{~nm}$ for (b) TE polarization and (c) TM polarization.
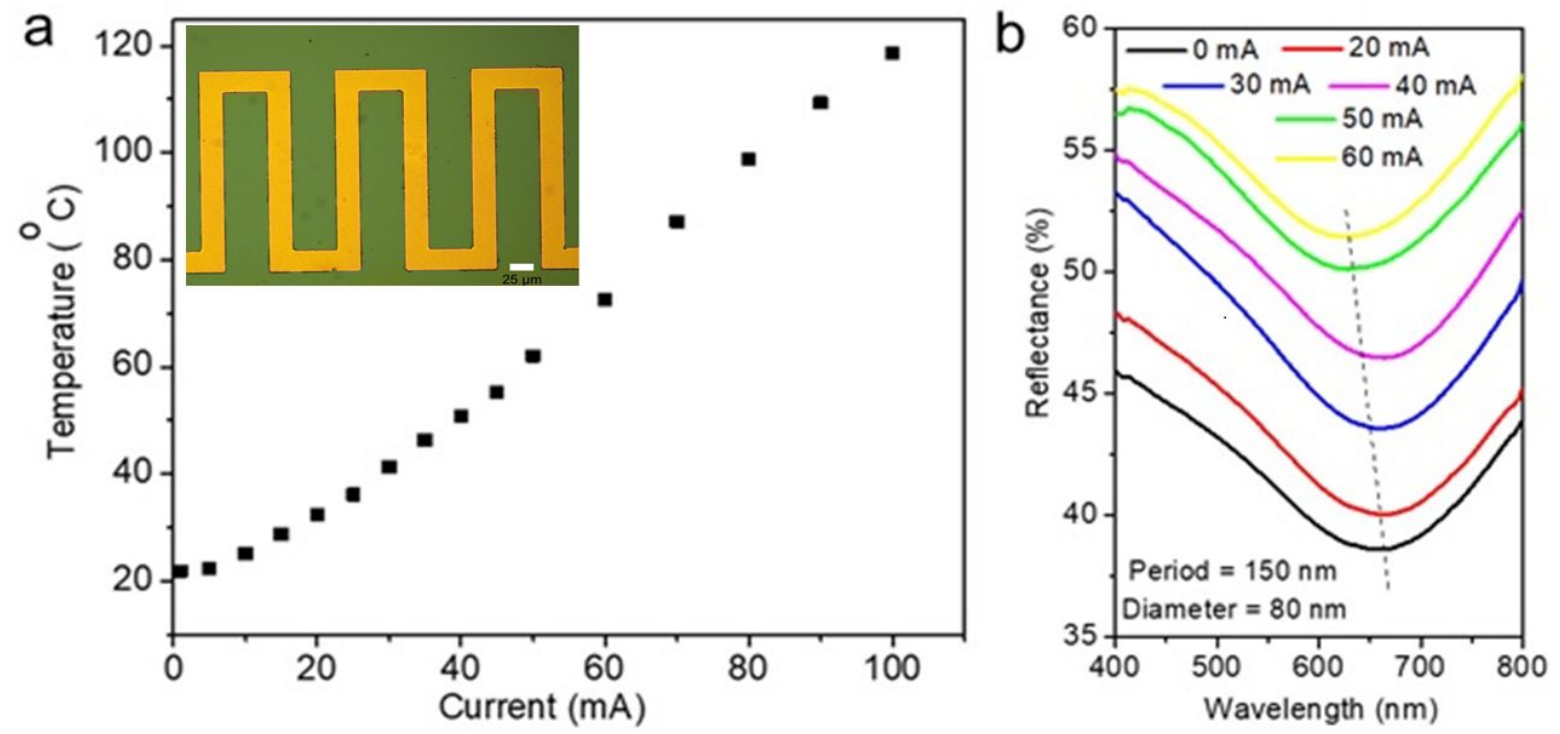

Figure S7. (a) Temperature calibration of microheater integrated $\mathrm{Sb}_{2} \mathrm{Te}_{3} / \mathrm{Si}$ device. Measured resistance (including contact resistance) of the device at room temperature is $749 \Omega$. Optical image of the heating element (width $=25 \mu \mathrm{m}$ ) is shown in the inset. (b) Measured reflectance spectra of 2D grating holes (hole diameter $=80 \mathrm{~nm}$ and period $=150 \mathrm{~nm}$ ) with applied current 